\title{
Hierárquicos, igualitários e contraditórios: posição social de adolescentes e orientação para a dominância social
}

\author{
Joao Wachelke \\ Fabíola Rodrigues Matos² \\ ${ }^{I}$ Instituto de Psicologia da Universidade Federal de Uberlândia, MG, Brasil \\ http://orcid.org/0000-0003-4364-8598 \\ ${ }^{2}$ Universidade Federal do Espírito Santo, ES, Brasil \\ http://orcid.org/0000-0002-2828-2869
}

\begin{abstract}
Resumo
O estudo avalia correlações entre concepções hierárquicas das relações entre grupos e as posições sociais de estudantes. O conceito de orientação à dominância social (SDO) aborda a aceitação de dominação e hierarquia entre grupos. A pesquisa teve 663 estudantes de Uberlândia-MG, com média de 16 anos de idade, que responderam um questionário com uma adaptação da Escala de Orientação à Dominância Social e questões referentes à escolaridade materna, sexo e renda familiar. Os resultados de análise de clusters apontam três padrões: adolescentes que defendem relações igualitárias entre os grupos; o padrão oposto, que aprova uma sociedade em que há dominantes e dominados; e um posicionamento contraditório, que aprova aspectos igualitários, mas nega que os grupos sejam equivalentes. A discussão centra-se nos fatores sexo e escolaridade e a sua associação com a SDO, e na interpretação do perfil contraditório que defende igualdade, mas restringe o seu escopo.
\end{abstract}

Palavras-chave: dominância, estrutura social, comportamento social, poder, grupos sociais.

Hierarchical, equal and contradictive: social position of adolescents and guidance for social dominance

\begin{abstract}
The study assesses correlations among hierarchical conceptions of group relationships and students' social position. The concept of social dominance orientation (SDO) deals with the acceptance of domination and hierarchy between groups. The research had 663 students from Uberlandia-MG, with a mean of 16 years of age who completed a questionnaire with an adaptation of the Social Dominance Orientation Scale and questions relative to mothers' schooling, sex and family income. Results from cluster analysis suggest three patterns: adolescents who support egalitarian relationships between groups; the opposite pattern, which approves of a society with dominant and dominated groups; and a contradictory position, which favors egalitarian aspects but denies that groups are equal. Discussion is centered on the sex and schooling factors and their association with SDO, and on the interpretation of the contradictory profile that defends restricted equality.
\end{abstract} Key words: dominance, social structure, social behavior, power, social groups. 


\section{Hierárquicos, igualitarios y contraditorios: posición social de los adolescentes y orientación para la dominancia social}

\section{Resumen}

El estudio evalúa correlaciones entre concepciones jerárquicas de las relaciones entre grupos y las posiciones sociales de estudiantes. El concepto de orientación a la dominancia social (SDO) aborda la aceptación de dominación y jerarquía entre grupos. La encuesta tuvo 663 estudiantes de Uberlandia-MG, con media de 16 años de edad que respondieron un cuestionario con una adaptación de la Escala de SDO y cuestiones referentes a la escolaridad materna, el sexo y la renta familiar. Un análisis de cluster apunta tres patrones: adolescentes que defienden relaciones igualitarias entre los grupos; el patrón opuesto, que aprueba una sociedad con dominantes y dominados; y un posicionamiento contradictorio, que aprueba aspectos igualitarios pero niega que los grupos sean equivalentes. La discusión se centra en los factores sexo y escolaridad y su asociación con la SDO, y en la interpretación del perfil contradictorio que defiende igualdad restringida.

Palabras clave: dominancia, estructura social, comportamiento social, poder, grupos sociales.

As relações entre grupos constituem tema clássico da Psicologia Social. São conhecidos os estudos de Sherif que demonstram que situações de competição entre grupos suscitam disposições mentais e comportamentais de rivalidade, bem como vieses de avaliar o grupo próprio como superior a outros grupos - como fez a escola de Bristol nos estudos de identidade social e relações intergrupais -, ou então basear-se em representações sobre a hierarquia social para avaliar grupos dominantes de forma positiva e grupos dominados negativamente, como sugerido pela linha de pesquisa do modelo de diferenciação categorial de Genebra (Amâncio, 2004; Torres \& Camino, 2013).

O presente trabalho busca avaliar a existência de perfis de respostas de estudantes secundaristas sobre concepções hierárquicas das relações entre grupos sociais, bem como as suas relações com as posições sociais que esses estudantes ocupam. A população escolar que cursa Ensino Médio diz respeito a cidadãos em formação, que pertencem a diversos segmentos da sociedade com hábitos, experiências e capacidades diferentes para interferir no mundo. Conhecer diferenças em representações ou códigos interpretativos dessas populações é importante, pois pode permitir avaliar a direção da socialização realizada e projetar as suas consequências para o convívio entre os grupos. Afinal, a adolescência é uma fase de transição em que se passa da influência quase exclusiva da família na infância para o contato com o mundo mais amplo de professores, amigos e outras fontes (Thomas \& Napolitano, 2017; Wachelke, 2018a).

Para dar conta da caracterização das relações entre grupos em termos de hierarquia e dominação, optamos por trabalhar com o fenômeno da orientação para a dominância social (social dominance orientation, SDO), proposto originalmente como construto psicológico (Sidanius \& Pratto, 1999). Nossa proposta é trabalhar com os indicadores (itens) do instrumento que avalia esse construto como sentenças que expressam proposições analisadas separadamente, de modo a analisar diferenças entre aspectos da hierarquia entre grupos conforme a posição social dos participantes. Cabe então apresentar brevemente o fenômeno.

A teorização sobre a orientação à dominância social desenvolveu-se através de estudos transculturais na década de 1990, buscando analisar aspectos psicológicos e sociais vinculados às formas de predisposição ao preconceito (Sidanius \& Pratto, 1999). O pressuposto fundamental se refere à concepção de que a sociedade tende a se estruturar em sistemas de grupos alicerçados em hierarquias sociais, havendo um pequeno número de grupos dominantes no topo e grupos subordinados na parte inferior (Heering \& Leone, 2018; Vala \& Lopes, 2016). Esses sistemas dispõem de alto grau de estabilidade, são difíceis de modificar e, em alguns casos, conseguem atenuar as desigualdades sociais (Cabral \& Oliveira, 2017; Giger, OrgambídezRamos, Gonçalves, Santos, \& Gomes, 2015). 
Sidanius e Pratto (1999) sugerem que uma sociedade organizada em hierarquias não categoriza os indivíduos por suas aptidões individuais, mas por suas pertenças sociais. Para a estruturação dessas hierarquias grupais existem três processos complementares: a discriminação individual, atos individuais de aceitação ou rejeição de indivíduos isolados por pertencerem a um grupo; a discriminação institucional, regras e ações de instituições que promovem distribuições assimétricas e desproporcionais de valores; e a assimetria comportamental, que diz respeito a diferenças de poder entre grupos e a comportamentos de dominância e submissão e correlatos (Fernandes \& Almeida, 2008; Santos \& Amâncio, 2014; Sidanius \& Pratto, 1999). Nesse sentido, as sociedades ainda apresentam sistemas de hierarquias nas relações intergrupais, que variam de acordo com a cultura e o contexto histórico, baseados na idade, no gênero e na arbitrariedade, sendo esse último relacionado a questões como a nacionalidade, a etnia, a religião, o poder, entre outros (Pratto, Sidanius, \& Levin; 2006; Sirbu, 2017).

A SDO reflete a oposição à igualdade entre grupos através de crenças, políticas sociais e afinidade por ideologias que mantenham a desigualdade (Ho et al., 2015; Sidanius \& Pratto, 1999). A meritocracia, por exemplo, é um modelo que reforça a hierarquia social, que reproduz uma ilusão de justiça, servindo à manutenção do status quo (Vala \& Lopes, 2016). Assim, a teoria de orientação à dominância social permite prever as relações intergrupais, sustentando essa percepção de igualdade ou a desigualdade entre grupos, sendo os pesquisadores dessa área responsáveis por identificar que mecanismos são encarregados de desenvolver e manter tais sistemas hierárquicos (Marques, 2016).

A SDO é geralmente considerada como um construto psicológico individual, que pode se expressar em níveis diferentes. Indivíduos com altos níveis de SDO tendem a favorecer ideologias e políticas que promovam as hierarquias, ao passo que indivíduos com níveis reduzidos têm maior tendência para políticas firmadas na igualdade social (Fernandes, Costa, Camino, \& Mendoza, 2007;
Giger et al., 2015; Sidanius \& Pratto, 1999). Assim, a orientação à dominância social é um dos preditores mais importantes do preconceito referido a diversos grupos em desvantagem social (Ekehammar \& Akrami, 2003; McFarland, 2010; Pratto, Sidanius, Stalworth, \& Malle, 1994). Também se correlaciona com uma vasta gama de atitudes sociais, ideológicas e comportamentos que promovem a desigualdade nas relações intergrupais, entre os quais o racismo, o sexismo, o elitismo cultural, a meritocracia e a ética protestante (Bates \& Heaven, 2001; Cima \& Dagallo, 2007; Jost \& Thompson, 2000; Pratto et al., 1994; Sidanius, Levin, \& Pratto, 1996; Sibley \& Duckitt, 2010; Sidanius, Levin, Liu, \& Pratto, 2000; Sidanius, Levin, Federico, \& Pratto, 2001; Valencia-Moya, 2018).

Em pesquisas recentes, relacionadas ao preconceito de forma geral, observa-se que a SDO é preditor significativo de homofobia, conforme estudo realizado com estudantes universitários da Nova Zelândia (O’Brien, Shovelton, \& Latner, 2013). Neste mesmo estudo, homens ainda apresentaram maiores níveis de SDO que mulheres (O’Brien et al., 2013). Na Itália, uma pesquisa demonstrou atitudes implícitas e explícitas sobre a obesidade em professores e estudantes de educação física, sendo que os professores relataram valores significativamente maiores no preconceito implícito e valores mais baixos na orientação à dominância social comparados aos estudantes (Gobbi, Greguol, Barboza Seron, \& Carraro, 2017). É observado também que os indivíduos que aceitam o acesso desigual à educação demonstram uma aceitação maior da estrutura hierárquica da sociedade, conforme pesquisa realizada com estudantes argentinos (Gatica, Martini, Dreizik, \& Imhoff, 2017).

No que concerne à temática da personalidade, Nicol e France (2016) indicaram que a abertura à experiência e a amabilidade são traços negativamente associados aos níveis de orientação à dominância social. A empatia está também associada negativamente à SDO, como foi indicado em amostra portuguesa (Crawley \& Suarez, 2016; Sirbu, 2017).

Há também resultados específicos que sustentam a influência de pais e pares nos níveis de orientação à do- 
minância social de adolescentes. Cross e Fletcher (2011) observaram que a responsividade dos pais está associada a menores níveis da orientação à dominância social, e que a conexão com grupos de alto status relaciona-se com altos níveis desse construto, assim como a importância de grupos de baixo status liga-se a visões mais igualitárias.

No Brasil, a orientação à dominância social foi diretamente associada aos valores materialistas e contrariamente associada aos valores pós-materialistas em uma amostra de estudantes universitários (Fernandes et al., 2007). Ainda no contexto brasileiro, tem-se que a SDO possui correlação positiva com racismo cordial (que geralmente se manifesta por meio de piadas de natureza racial) e racismo moderno (percepção de que os negros estão recebendo mais do que merecem e violando valores importantes para os brancos, por exemplo). A SDO também é considerada preditora do racismo moderno (Pires, 2010).

Visto isso, enfatiza-se a relevância da orientação à dominância social para pesquisas que envolvam as relações entre grupos, pois fornece conhecimento sobre como são as atitudes de uma determinada população perante hierarquias grupais. O presente trabalho visa a agregar conhecimento sobre a relação entre a posição ocupada por adolescentes na estrutura social e a sua aceitação ou rejeição em relação às sentenças vinculadas tematicamente à orientação para a dominância social.

Há grupos que dizem respeito à inserção diferenciada das pessoas em termos de acesso a recursos de poder para influenciar as próprias condições de vida. As classes sociais, geralmente categorizadas em termos de posse de recursos econômicos e culturais, são segmentações desse tipo. Nesse sentido, Bourdieu (1986) propõe a noção de capital, permitindo certa sobreposição com a noção de classe social a partir da posse ou não de riquezas, credenciais educacionais e culturais, redes de contatos e outros ativos sociais valiosos.

O estudo aqui relatado é uma investigação exploratória com o objetivo de caracterizar a adesão de adolescentes de Uberlândia (Minas Gerais) de posições sociais diferentes a sentenças a respeito da orientação para a dominação social. Por posição social, referimo-nos à classe econômica e escolaridade de seus pais, que refletem as condições de vida das famílias.

É possível explicar de maneira plausível tanto uma associação de crenças hierárquicas com posições privilegiadas ou desfavoráveis socialmente. No primeiro caso, pode se tratar de manutenção dos interesses do grupo e justificação da própria situação favorável, tal como em fenômenos como a justificação do sistema (Jost \& Banaji, 1994) e a crença no mundo justo (Lerner, 1980). Por outro lado, a associação com condições sociais mais precárias e a aprovação da hierarquia social poderia servir como motivação pessoal ao propor uma narrativa de vitoriosos e perdedores, levando pessoas em desvantagem a aguerrirem-se em busca dos objetivos; ou então servir como conscientização de privilégios próprios e da autocrítica por parte das posições favorecidas, ou até mesmo como hipocrisia ao rejeitar hierarquia, mas beneficiar-se dela objetivamente. No caso de escolaridade, o acesso a mais estudo poderia levar a concepções mais críticas acerca das relações entre grupos, com maior desaprovação à hierarquia social. Se existe lógica nas duas direções de interpretação, torna-se essencial observar os resultados empíricos antes de desenvolver hipóteses desse tipo.

\section{Método}

\section{Participantes}

Os participantes do estudo constituíram uma amostra de 663 estudantes matriculados na segunda série do Ensino Médio de três escolas de Uberlândia-MG. Houve equilíbrio entre os sexos, com 334 (40,3\%) participantes do sexo feminino. A média de idade foi de 16 anos ( $\mathrm{DP}=0,9)$, variando de 14 a 24 anos; 61,6\% tinham 16 anos de idade à época do estudo, e 95\% entre 15 e 17 anos. Em termos da proporção por escola, totais de 206 e 119 participantes (31,1\% e 17,9\%, respectivamente) estavam vinculados a duas escolas públicas, e 338 (51\%) a uma instituição particular. 
A escolaridade da mãe é preditor importante da escolaridade dos filhos, conforme Buchmann (2002), e por esse motivo essa informação foi tratada como dado de escolaridade da posição social. A maior parte dos participantes tinha mães que haviam completado o Ensino Superior (n $=264,40,2 \%)$ ou o Ensino Médio $(n=213,32,4 \%)$. Houve 101 participantes $(15,4 \%)$ com mães que concluíram até a 8. ${ }^{a}$ série, o Ensino fundamental 2, e 78 respondentes $(11,9 \%)$ cujas mães haviam estudado no máximo até a conclusão do Ensino Fundamental 1, ou 4. ${ }^{\mathrm{a}}$ série.

Estimamos a classe econômica dos participantes a partir de dados de escolaridade do chefe da família, acesso a bens de consumo e a serviços públicos no domicílio, por meio de adaptação do Critério de Classificação Econômica Brasil (CCEB, 2014) da Associação Brasileira de Empresas de Pesquisa (ABEP, 2014). Consideramos as classes econômicas A (renda superior), B1, B2 e C (renda inferior). Em 2014, conforme dados do CCEB (2014), a classe A tinha renda média familiar mensal de R $\$ 11.037$, a classe B1 tinha renda média de $\mathrm{R} \$ 6.006$, a classe $\mathrm{B} 2$ renda de $\mathrm{R} \$ 3.118$ e a classe $\mathrm{C}$ compreendia subclasses $\mathrm{C} 1$, com renda média de $\mathrm{R} \$ 1.865$, e $\mathrm{C} 2$, com média de $\mathrm{R} \$ 1.277$. A maior parte dos participantes era de classe econômica $\mathrm{A}(\mathrm{n}=$ $239,37,7 \%)$, seguidos pelas classes B2 $(n=158,24,9 \%)$, B1 $(n=120,18,9 \%)$ e C $(n=117,18,5 \%)$.

\section{Instrumento}

Foi empregado um questionário autoaplicado com seções sobre opiniões dos respondentes sobre as relações sociais e uma adaptação da Escala de Orientação à Dominância Social de Fernandes et al. (2007) que, por sua vez, consiste em versão validada para o português brasileiro de instrumento original de Sidanius e Pratto (1999). A medida apresenta dezesseis sentenças a respeito das relações entre grupos, metade das quais expressa apoio e metade divergências com a orientação à dominação social como, por exemplo: item 4 - "para progredir na vida, às vezes, é necessário pisar nos outros grupos" (apoio) e item 13 - "deveríamos aumentar a igualdade social" (divergência). A medida validada é na forma de escala Likert de 7 pontos, de totalmente em desacordo a totalmente de acordo. No entanto, neste estudo optamos por ter cinco opções de resposta: "discordo"; "discordo, mas não muito"; "concordo, mas não muito"; "concordo"; e “?”, no caso de dificuldade de posicionar-se. A alteração justifica-se pela intenção de ter um questionário de mais simples compreensão para a população escolar.

Também havia no instrumento questões sociodemográficas (idade, sexo, escolaridade dos pais, acesso a bens de consumo, serviços no domicílio e outros). Essa última seção foi uma adaptação do Critério de Classificação Econômica Brasil de 2015, lançado em 2014 (ABEP, 2014).

\section{Procedimento}

O projeto foi aprovado pelo Comitê de Ética da Universidade Federal de Uberlândia, com o parecer n. ${ }^{\circ}$ 379.510. Após autorização da direção das escolas participantes, a coleta de dados ocorreu no segundo semestre do ano de 2014, com o recolhimento em ocasião prévia dos termos de consentimento assinados pelos cuidadores dos estudantes.

A coleta de dados foi realizada em horário de aulas. A equipe de pesquisa foi formada pelo autor principal e três assistentes de pesquisa treinados. Cada situação de coleta teve pelo menos duas pessoas responsáveis, sendo o autor principal uma delas. Após instruções coletivas, os estudantes tiveram 45 minutos para responder ao questionário individualmente. As dúvidas de entendimento da atividade foram resolvidas prontamente pela equipe.

A análise de dados visou a identificar padrões multivariados de resposta às sentenças sobre orientação para a dominação social. Desse modo, escolhemos a análise de clusters (Kassambara, 2017), que permite classificar grupos de participantes a partir da distância entre as respostas que fornecem. As análises foram realizadas no ambiente de programação R (R Core Team, 2019), com auxílio dos pacotes cluster (Maechler, Rousseuw, Struyf, Hubert, \& Hornik, 2018) e factoextra (Kassambara \& Mundt, 2017). 
A análise foi realizada sobre as respostas dos itens da Escala de Orientação à Dominância Social, considerando-se duas categorias: "concordo", a fusão das respostas "concordo" e "concordo, mas não muito", e "discordo", fusão das repostas de discordância. O agrupamento respaldou-se na identificação da tendência qualitativa de concordância ou discordância, admitindo-se discrepâncias ou estilos de resposta diferentes. As respostas "?" foram consideradas como dados omissos; para dois itens elas tiveram proporção de $2 \%$, mas para doze das sentenças elas não ultrapassaram $1 \%$. Contudo, todos os participantes foram contemplados na análise, já que os procedimentos empregados toleram dados incompletos.

\section{Resultados}

Primeiro foi calculada a adequação dos dados para a análise de clusters, por meio da estatística de Hopkins.
Com o valor de 0,14 , inferior a 0,50 , os dados indicaram a existência de agrupamentos. A distância de Gower (1971), compatível com dados nominais, foi a medida de dissimilaridade utilizada entre os casos. Os clusters foram obtidos pelo método aglomerativo da distância de Ward, por meio do procedimento daisy do pacote hclust.

Após estudo de índices como tamanho dos clusters e homogeneidade intra e interclusters, optou-se por trabalhar com três agrupamentos. O cluster 1 teve 257 participantes, o cluster 2 teve aproximadamente metade da amostra, com 331, e o cluster 3 representou apenas 11\% dos participantes, com 75 .

A Tabela 1 apresenta as proporções das respostas aos itens da medida adaptada de orientação à dominação social. Na parte de baixo há a composição demográfica de cada cluster em termos de classe econômica, escolaridade da mãe dos participantes e sexo.

Tabela 1. Clusters formados a partir de respostas percentuais de concordância $(\% \mathrm{C})$ e discordância $(\% \mathrm{D})$ a itens sobre orientação à dominação social.

\begin{tabular}{|c|c|c|}
\hline & \multicolumn{2}{|c|}{ Cluster 1} \\
\hline & $C \%$ & $D \%$ \\
\hline 1. Alguns grupos têm, simplesmente, mais valor do que outros. & 23 & 77 \\
\hline $\begin{array}{l}\text { 2. Ao fazer o que o grupo quer, às vezes é necessário usar a força contra outros } \\
\text { grupos. }\end{array}$ & 12 & 88 \\
\hline 3. Os grupos superiores devem dominar os grupos inferiores. & 2 & 98 \\
\hline 4. Para progredir na vida, às vezes é necessário pisar nos outros grupos. & 3 & 97 \\
\hline $\begin{array}{l}\text { 5. Se certos grupos permanecessem em seu devido lugar, teríamos menos } \\
\text { problemas. }\end{array}$ & 36 & 64 \\
\hline 6. Provavelmente é bom que alguns grupos fiquem em cima e outros embaixo. & 7 & 93 \\
\hline 7. Os grupos inferiores devem permanecer em seu lugar. & 3 & 97 \\
\hline 8. Em certas ocasiões outros grupos devem ser mantidos em seu lugar. & 36 & 64 \\
\hline 9. Seria bom que todos os grupos pudessem ser iguais. & 83 & 17 \\
\hline 10. A igualdade entre os grupos deve ser o nosso ideal. & 79 & 21 \\
\hline
\end{tabular}

\begin{tabular}{|c|c|}
\hline \multicolumn{2}{|c|}{ Cluster 2} \\
\hline$C \%$ & $D \%$ \\
\hline 66 & 34 \\
\hline 76 & 24 \\
\hline 20 & 80 \\
\hline 16 & 84 \\
\hline 73 & 27 \\
\hline 39 & 61 \\
\hline 26 & 74 \\
\hline 68 & 32 \\
\hline 94 & 6 \\
\hline 94 & 6 \\
\hline
\end{tabular}

\begin{tabular}{|c|c|}
\hline \multicolumn{2}{|c|}{ Cluster 3} \\
\hline$C \%$ & $D \%$ \\
\hline 68 & 32 \\
\hline 79 & 22 \\
\hline 42 & 58 \\
\hline 52 & 48 \\
\hline 70 & 30 \\
\hline 75 & 25 \\
\hline 49 & 51 \\
\hline 84 & 16 \\
\hline 8 & 92 \\
\hline 5 & 95 \\
\hline
\end{tabular}




\begin{tabular}{|c|c|c|c|c|c|c|}
\hline & \multicolumn{2}{|c|}{ Cluster 1} & \multicolumn{2}{|c|}{ Cluster 2} & \multicolumn{2}{|c|}{ Cluster 3} \\
\hline & $C \%$ & $D \%$ & $C \%$ & $D \%$ & $C \%$ & $D \%$ \\
\hline 11. Todos os grupos devem ter as mesmas oportunidades na vida. & 97 & 4 & 95 & 5 & 68 & 32 \\
\hline $\begin{array}{l}\text { 12. Teríamos menos problemas se tratássemos os grupos diferentes de forma } \\
\text { igualitária. }\end{array}$ & 93 & 7 & 90 & 10 & 60 & 40 \\
\hline 13. Deveríamos aumentar a igualdade social. & 98 & 2 & 97 & 3 & 67 & 33 \\
\hline $\begin{array}{l}\text { Devemos fazer o que for possível para igualar as condições dos vários grupos } \\
\text { sociais. }\end{array}$ & 96 & 4 & 97 & 3 & 51 & 49 \\
\hline 15. Devemos nos esforçar para tornar os salários ou remunerações mais iguais. & 85 & 15 & 93 & 7 & 46 & 54 \\
\hline 16. Nenhum grupo deve dominar na sociedade. & 85 & 15 & 86 & 14 & 27 & 73 \\
\hline \multicolumn{7}{|l|}{ SEXO } \\
\hline Masculino & \multicolumn{2}{|c|}{$97(38 \%)$} & \multicolumn{2}{|c|}{$176(53 \%)$} & \multicolumn{2}{|c|}{$56(75 \%)$} \\
\hline Feminino & \multicolumn{2}{|c|}{$160(62 \%)$} & \multicolumn{2}{|c|}{$155(47 \%)$} & \multicolumn{2}{|c|}{$19(25 \%)$} \\
\hline \multicolumn{7}{|l|}{ CL. ECONÔMICA } \\
\hline$A$ & \multicolumn{2}{|c|}{$115(47 \%)$} & \multicolumn{2}{|c|}{$94(30 \%)$} & \multicolumn{2}{|c|}{$30(42 \%)$} \\
\hline$B 1$ & \multicolumn{2}{|c|}{$45(18 \%)$} & \multicolumn{2}{|c|}{$57(18 \%)$} & \multicolumn{2}{|c|}{$18(25 \%)$} \\
\hline$B 2$ & \multicolumn{2}{|c|}{$51(21 \%)$} & \multicolumn{2}{|c|}{$95(30 \%)$} & \multicolumn{2}{|c|}{$12(17 \%)$} \\
\hline C & \multicolumn{2}{|c|}{$33(14 \%)$} & \multicolumn{2}{|c|}{$72(23 \%)$} & \multicolumn{2}{|c|}{$12(17 \%)$} \\
\hline \multicolumn{7}{|l|}{ ESCOLARIDADE } \\
\hline Fundamental 1 & \multicolumn{2}{|c|}{$19(7 \%)$} & \multicolumn{2}{|c|}{$52(16 \%)$} & \multicolumn{2}{|c|}{$7(9 \%)$} \\
\hline Fundamental 2 & \multicolumn{2}{|c|}{$26(10 \%)$} & \multicolumn{2}{|c|}{$65(20 \%)$} & \multicolumn{2}{|c|}{$10(14 \%)$} \\
\hline Médio & \multicolumn{2}{|c|}{$83(33 \%)$} & \multicolumn{2}{|c|}{$95(29 \%)$} & \multicolumn{2}{|c|}{$35(47 \%)$} \\
\hline Superior & \multicolumn{2}{|c|}{$126(50 \%)$} & \multicolumn{2}{|c|}{$116(35 \%)$} & 22( & $\%)$ \\
\hline $\mathrm{N}$ & & & & & & \\
\hline
\end{tabular}

Para descrição dos clusters, é pertinente lembrar que os oito primeiros indicadores têm conteúdo que expressam concordância com a orientação para a dominância social, enquanto os indicadores de número 9 a 16 expressam o oposto, isto é, uma visão igualitária. O cluster 1 reúne participantes em proporção expressiva na amostra, com maior representação do sexo feminino. A escolaridade das mães e a classe econômica dos participantes também tem modalidades mais elevadas que a do perfil geral da amostra. As respostas desse agrupamento são marcadas por rejeição à dominância social e aprovação de relações igualitárias, com maioria das respostas compatíveis com esse padrão em todos os itens.

O cluster 2, que diz respeito a quase metade dos participantes, diverge do primeiro na medida em que apoia algumas sentenças alinhadas com a dominância 
social: tende a reconhecer mais valor em alguns grupos que outros (item 1), admite ser necessário o uso de força contra grupos (item 2), que se grupos inferiores fossem mantidos no lugar haveria menos problemas (item 5), e que em certas ocasiões alguns grupos deveriam ser mantidos em seu lugar (item 8). Porém também apoiam com ampla maioria as sentenças que defendem igualdade nas relações. Esse padrão envolve, portanto, contradições aparentes entre o apoio simultâneo à igualdade e à hierarquia. A composição por sexo é similar a da amostra geral quanto ao sexo, mas a classe econômica e escolaridade materna são inferiores.

Por fim, o cluster 3, minoritário como padrão, tem três quartos de participantes homens, perfil econômico ligeiramente inferior ao da amostra global, e escolaridade da mãe concentrada no Ensino Médio completo. Esse agrupamento tem preferência por relações hierárquicas, concordando nitidamente com cinco das sentenças pró-SDO e com proporções divididas nas outras três, e discordando majoritariamente de que seria bom se todos os grupos fossem iguais (item 9), que a igualdade entre os grupos deve ser um ideal (item 10) e que nenhum grupo deve dominar (item 16). Há respostas divididas em sentenças acerca de condições e rendimentos iguais dos grupos (itens 14 e 15) e concordância somente com os itens igualitários restantes. Desse modo, ainda que exista apoio a algumas proposições igualitárias, a tendência dominante de metade dos itens respondidos é pela hierarquia, e há respostas equilibradas em outros cinco.

\section{Discussão}

Os resultados apontam três padrões de resposta na amostra de estudantes uberlandenses: adolescentes que defendem relações igualitárias entre os grupos e repudiam algum tipo de dominação; o padrão oposto, que sugere a aprovação de uma visão da sociedade em que há dominantes e dominados, justificando uso de força e reservando lugares sociais específicos a ambos; e um posicionamento aparentemente contraditório, que aprova aspectos igualitários e simultaneamente nega que todos os grupos mereçam ser considerados iguais. Para fins de clareza na apresentação, chamaremos ao primeiro padrão perfil igualitário, ao segundo perfil hierárquico e ao terceiro, contraditório.

Na investigação relatada em particular, o perfil contraditório foi minoritário, mas não se justifica inferir que assim o seja empiricamente na população; o estudo teve caráter exploratório e amostragem não probabilística. Devido ao tamanho razoável da amostra, contudo, podemos afirmar alguma estabilidade nos dados. A presente discussão volta-se preferencialmente para a interpretação de cada perfil, da lógica social que lhes é subjacente e da avaliação da relação de tendências referentes à orientação para a dominância social com fatores de posição social.

Duas coordenadas sociais destacam-se quanto à importância em termos de um posicionamento mais ou menos hierárquico. Primeiramente, o sexo dos participantes: estudantes do sexo feminino predominaram no perfil igualitário e, inversamente, adolescentes do sexo masculino foram três quartos do perfil hierárquico. A orientação para a dominância social tende a ser maior para homens em pesquisas de diversas culturas documentadas na literatura (Fraser, Osborne, \& Sibley, 2015; O’Brien et al., 2013; Sidanius, Sinclair, \& Pratto, 2006). Diferenças sistemáticas entre os gêneros apontam para a importância de avaliação da interação de fatores biológicos e investimento social que resultam em papeis sociais opostos de homens e mulheres, essas marcadas pela expressividade e cuidado e aqueles pela instrumentalidade e poder, como sugerido por teorias como a dos papeis sexuais de Eagly e Wood (2012). Não se pretende aqui explicar com quaisquer indícios de causalidade para as diferenças encontradas, mas constatar que também no estudo conduzido foram encontradas diferenças importantes de associação de padrões de resposta com os sexos, corroborando a literatura e reforçando tendência sistemática. 
A escolaridade materna também parece ter importância em relação com a aceitação da orientação à dominância social. O perfil igualitário tem proporção maior de adolescentes com mães com nível superior, enquanto o perfil hierárquico tem concentração que se destaca de participantes com mães com Ensino Médio. Carvacho et al. (2013) identificaram em amostras europeias e sul-americanas que a escolaridade e a renda têm relações negativas com a orientação para a dominância social, e que o efeito da escolaridade é mais importante. No presente estudo, condição econômica não teve relações fortes com os padrões identificados. As representações associadas à posição cultural marcada pela escolaridade tiveram associações mais nítidas.

A escolaridade também não teve relação linear: a visão hierárquica está mais ligada aos participantes com mães de escolaridade até Ensino Médio. Não há dados para avaliar outros aspectos como trajetória familiar em termos de mobilidade social intergeracional, mas possivelmente para pessoas em situação econômica desfavorável uma estratégia pautada no esforço individual pode se mostrar - ou tenha se mostrado em gerações ou períodos anteriores - eficaz para investimento na educação dos filhos e manutenção de certas práticas de trabalho que levem à ascensão na pirâmide da sociedade. Se, como indicado na literatura, a meritocracia está associada à orientação para a dominância social, uma percepção da sociedade com vencedores e perdedores também pode implicar uma clivagem entre grupos superiores e inferiores, e refletir na busca pelo pertencimento aos primeiros. Nesse ponto há um paralelo com estudos realizados com coleta em 2013, na mesma cidade de Uberlândia, com amostra semelhante de estudantes: constatou-se forte adesão por estudantes de posição social desfavorável - incluindo a escolaridade materna - quanto a fenômenos relacionados teoricamente com a orientação para a dominância social, como a meritocracia e o apoio a hierarquias de trabalho (Wachelke, 2017) e a crença de que o mundo é justo, também entendido como ideologia conservadora (Wachelke, 2018a).
O perfil contraditório apresenta uma realidade que demanda maior aprofundamento de estudos futuros. No entanto, há possibilidades interpretativas. Valores como a igualdade são valorizados socialmente, que faz com que sejam endossados, ao menos em discursos explícitos, com frequência, como observado em estudo com amostras similares (ver Wachelke, 2018b). Por terem conotação positiva e civilizatória, a sua rejeição é improvável; conforme Freeden (2015), a igualdade é de um dos pilares do liberalismo, ideologia moderna e multifacetada que predomina nos tempos atuais. Rejeitar a igualdade geraria certo estranhamento mas há quem o faça, como no perfil hierárquico, aqui minoritário. No entanto, a interpretação do que seja igualdade ou o seu escopo podem sofrer variações. Pode-se fazer analogia, por exemplo, com o que ocorre com a justiça. Poucos diriam prezar pela injustiça, mas há modos diferentes de conceber o que é justiça, como apontam contribuições como as de Deutsch (1975), que diferenciam entre justiça como igualdade, equidade e necessidade. Além disso, as pessoas frequentemente reservam preocupações com justiça não para todos, mas somente a pessoas que estejam incluídas em certos critérios, dentro de certas fronteiras psicossociais, por exemplo, pessoas que compartilham uma identidade social ou certos valores, como demonstram Lima Nunes, Pereira e Correia (2013): trata-se da noção de escopo de justiça. Possivelmente a contradição inicialmente identificada no respectivo perfil da presente investigação seja explicada por fenômeno semelhante: os participantes se percebem como igualitários - e por que não dizer, em certo sentido, justos - e defendem isso, mas simultaneamente entendem que a igualdade deve ser aplicada somente a certos grupos ou segmentos da sociedade, e não a todos. Seria uma espécie de igualdade seletiva, um modo de aderir ao menos parcialmente à justificação de dominação entre grupos. Assumindose essa postura interpretativa, o mais preciso seria tratar desse perfil como um padrão ambivalente, em vez de propriamente contraditório. Não se trata de 
uma contradição no sentido da lógica formal clássica (ver Aristóteles, 2016), isto é, da impossibilidade de duas proposições serem simultaneamente verdadeiras, mas sim de especificação implícita de que igualdade e hierarquia são aceitas, porém associadas a contextos de extensão diferente: são respectivamente relações válidas para alguns grupos de status superior, ou para o conjunto de todos os grupos. Outra possibilidade seria a de mascarar uma adesão latente que se manifeste em outros contextos, mas que implique riscos para a autoimagem ou desejabilidade social, haja vista que o apoio à dominância social possa ser repreensível. São pontos a serem avaliados por estudos subsequentes, capazes de verificar a existência de processos psicossociais compatíveis com essas hipóteses.

É pertinente lembrar que a amostra de 2014 teve adolescentes, hoje na idade adulta. A orientação para a dominância social na adolescência pode ter peculiaridades e diferenciar-se do que é observado nas pesquisas, a maior parte realizada junto a universitários ou adultos. $\mathrm{O}$ estudo de Cornelis, Van Hiel, Roets e Kossowka (2008) identificou algumas mudanças de pequena magnitude com a idade em uma amostra polonesa, com correlação negativa da SDO com a idade, mas não em uma amostra belga. Jetten e Iyer (2010) não observaram diferença significativa em escores de SDO em um estudo longitudinal cobrindo pouco mais de um semestre na vida de estudantes universitários britânicos. Contudo, há evidências de que valores se modificam durante o ciclo de vida (ver Gouveia, Vione, Milfont, \& Fischer, 2015), e há correlações entre valores e SDO (Fernandes et al., 2007). Ademais, é importante ressaltar a natureza não probabilística da amostra, o que demandaria replicações da pesquisa ou realização de investigações semelhantes em outros contextos para avaliar se os padrões identificados encontram respaldo mais amplo. Portanto, cabe não generalizar indevidamente os resultados para outros contextos e etapas da vida.

Ainda no que diz respeito às limitações do estudo, é preciso associar os resultados às condições da pesquisa, isto é, do contexto nacional de 2014: pesquisas sociais como a realizada refletem o ambiente que lhes deu origem e que permite explicá-las. Frente ao dinamismo veloz da situação política e social brasileiras desde então, parece prudente não imaginar que esses padrões se mantenham necessariamente. A natureza exploratória do estudo também exige clareza na leitura acerca das restrições às interpretações dos processos explicativos ligados aos resultados: dentro das características dos levantamentos não probabilísticos, não há evidências indutivas ou dedutivas desses processos, mas tão somente formulação de explicações hipotéticas para eventual refinamento e verificação por pesquisas futuras.

É necessário também comentar a ausência de diferenças relacionadas à renda dos participantes. Apesar do caráter exploratório da pesquisa, trabalhamos com a expectativa de encontrar condições de inteligibilidade da composição dos perfis na posição social dos estudantes. Em estudos clássicos como A Distinção, de Bourdieu (1979), há homologia entre o volume de capital econômico e preferências e opiniões de participantes. No entanto, configurações sociais em termos de relações entre grupos diferentes, temáticas de interesse diversos, e contextos distintos quanto a períodos temporais e abrangência cultural implicam relações de mediação entre opiniões e inserção social também distintas. Neste caso, houve contrastes em uma das variáveis analisadas de posição social, que também corresponde a uma operacionalização de classe social, o capital cultural ou escolaridade materna. A renda ou classe econômica distribuiu-se de maneira pouco diferenciada entre os perfis. Para as crenças de adolescentes sobre relações entre grupos, no contexto cultural e temporal analisado, há alguma capacidade explicativa a respeito de seu contato com certos conhecimentos culturais escolaridade -, e menos importância da consideração isolada do acesso a bens de consumo e serviços, a que correspondeu a operacionalização de classe econômica.

Uma opção do estudo realizado que a nosso ver indica sutilezas interessantes está na avaliação da orien- 
tação para a dominância social em termos de sentenças separadas. A maior parte dos estudos trata o construto como traço latente inferido indiretamente a partir de itens de escalas psicométricas, gerando um escore único sustentado por medidas de validade e confiabilidade. No entanto, a estratégia empregada no presente estudo apontou para padrões de resposta distintos da SDO que poderiam justificar algumas investigações do comportamento de resposta às sentenças isoladamente, levando a constatações e ideias para estudos futuros pertinentes.

Finalmente, após identificar os perfis - por que não dizer, ideológicos? - de aprovação de hierarquia ou igualdade entre os grupos por meio da orientação para a dominância social, caberá uma análise não propriamente científica, mas ética, a respeito de suas implicações para a sociedade brasileira. Se as Ciências Sociais, como sustenta Flyvbjerg (2001), devem fornecer subsídios para decidir os rumos da sociedade, existirá algum perfil mais aconselhável para o entendimento das relações entre grupos? Será melhor cultivar a igualdade ou uma hierarquia competitiva? Particularmente, concordamos com a primeira alternativa. A promoção desse tipo de cultura ética, e por ética queremos dizer referente a normas e modelos de conduta, passa por uma análise necessária da cultura e dos padrões de socialização que legam papeis a homens e mulheres - veja-se por exemplo a ideologia da dominação masculina (Bourdieu, 2002), já que há nítida relação entre a preferência dos sexos por um ou outro perfil. Além disso, a maior escolaridade materna associando-se à igualdade provavelmente reflete maior sofisticação formal e conhecimento de cultura humanística propiciados por percurso educacional prolongado, que se reflete na socialização dos filhos. Se, refletindo tendências recentes, passa-se a cultivar a hierarquia explicitamente em termos de posicionamentos oficiais ou mesmo de mudanças em conteúdos curriculares escolares, pode-se reforçar uma tendência inversa. São questões levantadas também pela investigação aqui realizada, mas cujo aprofundamento ultrapassa nossos objetivos.

\section{Referências}

Associação Brasileira de Empresas de Pesquisa. (2014). Critério de Classificação Econômica Brasil. Alterações na aplicação do Critério Brasil, válidas a partir de 01/01/2015. Acesso em 26 março 2019 em http://www.abep.org. https://doi.org/10.11606/d.96.2010. tde-13122010-084622

Aristóteles. (2016). Órganon. $3^{a}$ ed. São Paulo: Edipro.

Amâncio, L. (2004). Identidade social e relações intergrupais. In J. Vala, M. B. Monteiro (Eds.), Psicologia social (pp. 387-409). Lisboa, Portugal: Fundação Calouste Gulbenkian.

Bates, C., \& Heaven, P. C. (2001). Attitudes to women in society: The role of social dominance orientation and social values. Journal of Community \& Applied Social Psychology, 11,43-49. https://doi.org/10.1002/casp.589

Bourdieu, P. (1979). La distinction: critique sociale $d u$ jugement. Paris: Minuit.

Bourdieu, P. (1986). The forms of capital. In J. Richardson (Ed.), Handbook of theory and research for the sociology of education (pp. 241-258). Westport, EUA: Greenwood.

Bourdieu, P. (2002). A dominação masculina (2a ed.). Rio de Janeiro, BR: Bertrand Brasil.

Buchmann, C. (2002). Measuring family background in international studies of education: conceptual issues and methodological challenges. In A. Porter, A. Gamoran (Ed.), Methodological advances in cross-national surveys of educational achievement (pp. 150-197). Washington, EUA: National Academy Press. https://doi.org/10.17226/10322

Cabral, M. L., \& Oliveira, I. (2017). Trauma social em idade avançada. Revista Saúde e Pesquisa, 10(3), 485-492. https://doi.org/10.17765/1983-1870.2017v$\underline{10 n 3 p 485-492}$

Carvacho, H., Zick, A., Haye, A., González, R., Manzi, J., Kocik, C., \& Bertl, M. (2013). On the relation between social class and prejudice: the roles of education, income, and ideological atitudes. European Journal of Social Psychology, 43, 272-285. https://doi.org/10.1002/ejsp.1961 
Cima, R., \& Dallago, F. (2007). Existe una correlación negativa entre el autoritarismo de derechas y la orientación a la dominancia social. Psicologia Politica, 34, 79-97. (DOI INEXISTENTE)

Cornelis, I., Van Hiel, A., Roets, A., \& Kossowka, M. (2008). Age differences in conservatism: evidence on the mediating effects of personality and cognitive style. Journal of Personality, 77(1), 51-88. https://doi. org/10.1111/j.1467-6494.2008.00538.x

Crawley, D., \& Suarez, R. (2016). Empathy, Social Dominance Orientation, Mortality Salience, and Perceptions of a Criminal Defendant. SAGE Open, 6(1), 1-15. https://doi.org/10.1177/2158244016629185

Cross, J. R., \& Fletcher, K. L. (2011). Associations of parental and peer characteristics with adolescents' social dominance orientation. Journal of Youth Adolescence, 40(6), 694-706. https://doi.org/10.1007/s10964-010-9585-7

Deutsch, M. (1975). Equity, equality, and need: what determines which value will be used as the basis of distributive justice? Journal of Social Issues, 31(3), 137149. https://doi.org/10.1111/j.1540-4560.1975.tb01000.x

Eagly, A. H., \& Wood, W. (2012). Social role theory. In P. A. M. Van Lange, A. W. Kruglanski, \& E. T. Higgins (Eds.), Handbook of theories of social psychology (pp. 458-476). Thousand Oaks: Sage. https:// doi.org/10.4135/9781446249222.n49

Ekehammar, B., \& Akrami, N. (2003). The relation between personality and prejudice: A variable- and a person-centred approach. European Journal of Personality, 17, 449- 464. https://doi.org/10.1002/per.494

Fernandes, S. C., \& Almeida, S. S. (2008). Mensuração e análise dos níveis de orientação à dominância social. Psicologia em Foco, 1(1), 1-7.

Fernandes, S., Costa, J., Camino, L., \& Mendoza, R. (2007). Valores psicossociais e orientação à dominância social: Um Estudo Acerca do Preconceito. Psicologia: Reflexão e Crítica, 20(3), 490-498. https:// doi.org/10.1590/s0102-79722007000300017

Flyvbjerg, B. (2001). Making social science matter. Why social inquiry fails and how it can succeed again. Nova Iorque, EUA: Cambridge University Press. https://doi. org/10.1017/cbo9780511810503
Fraser, G., Osborne, D., \& Sibley, C. G. (2015). "We want you in the workplace, but only in a skirt!" Social dominance orientation, gender-based affirmative action and the moderating role of benevolent sexism. Sex Roles, 73(5), 231-244. https://doi.org/10.1007/s11199-015-0515-8

Freeden M. (2015). Liberalism: a very short introduction. Oxford, EUA: Oxford University Press. (DOI INEXISTENTE)

Gatica, L., Martini, J. P., Dreizik, M., \& Imhoff, D. (2017). Psychosocial and psycho-political predictors of social inequality justification. Psicología, 35(1), 279-310.

Giger, J., Orgambídez-Ramos, A., Gonçalves, G., Santos, J., \& Gomes, A. (2015). Evidências métricas da adaptação da Escala de Dominância Social numa amostra portuguesa. Avaliação Psicológica, 14(1), 143-151. https://doi.org/10.15689/ap.2015.1401.16

Gobbi, E., Greguol, M., Barboza Seron, B., \& Carraro, A. (2017). Um estudo exploratório da tendência "antiobesidade" entre professores e estudantes de educação física italianos. Movimento, 23(3), 963-974. https://doi. org/10.22456/1982-8918.73193

Gouveia, V. V., Vione, K. C., Milfont, T. L., \& Fischer, R. (2015). Patterns of value change during the life span: some evidence from a functional approach to values. Personality and Social Psychology Bulletin, 41(9), 1276-1290. https://doi.org/10.1177/0146167215594189

Gower, J. C. (1971). A general coefficient of similarity and some of its properties. Biometrics, 27(4), 857-871. https://doi.org/10.2307/2528823

Heering, M. S., \& Leone, L. (2018 - no prelo). Power Moderates the Effects of Social Dominance Orientation on Punishment: An Experimental Analysis. Psychological Reports. https://doi.org/10.1177/0033294118755095

Ho, A. K., Sidanius, J., Kteily, N., Sheehy-Skeffington, J., Pratto, F., Henkel, K. E., \& Stewart, A. L. (2015). The Nature of Social Dominance Orientation: Theorizing and Measuring Preferences for Intergroup Inequality Using the New SDO7 Scale. Journal of Personality and Social Psychology, 109(6), 1003-1028. https://doi. org/10.1037/pspi0000033

Jetten, J., \& Iyer, A. (2010). Different meanings of the social dominance orientation concept: predicting political attitudes over time. British Journal of Social Psychology, 49, 385-404. https://doi.org/10.1348/014466609x435723 
Jost, J. \& Banaji, M. R. (1994). The role of stereotyping in system-justification and the production of false consciousness. British Journal of Social Psychology, 33, 1-27. https://doi.org/10.1111/j.2044-8309.1994.tb01008.x

Jost, J. T., \& Thompson, E. (2000). Groupbased dominance and opposition to equality as independent predictors of self-esteem, ethnocentrism, and social policy attitudes among African Americans and European Americans. Journal of Experimental Social Psychology, 36, 209-232. https://doi.org/10.1006/jesp.1999.1403

Kassambara, A. (2017). Practical guide to cluster analysis in R: unsupervised machine learning. Marseille, Fr: STHDA.

Kassambara, A., \& Mundt, F. (2017). factoextra: Extract and Visualize the Results of Multivariate Data Analyses. R package version 1.0.5. Recuperado de https://CRAN.Rproject.org $/$ package $=$ factoextra

Lerner, M. J. (1980). The belief in a just world: a fundamental decision. Nova Iorque, EUA: Springer. https:// doi.org/10.1007/978-1-4899-0448-5

Lima Nunes, A. V., Pereira, C. R., \& Correia, I. (2013). Justice seems not to be for all: exploring the scope of justice. In-Mind, 17(2). Recuperado de http://www. in-mind.org/article/justice-seems-not-to-be-for-all-exploring-the-scope-of-justice

Maechler, M., Rousseeuw, P., Struyf, A., Hubert, M., \& Hornik, K. (2018). Cluster: Cluster analysis basics and extensions. R package version 2.0.7-1.

Marques, S. C. B. (2016). Vulnerabilidade percebida à doença e movimentos de migração: A crise de refugiados e as suas implicações para o sistema imunitário comportamental (Dissertação de mestrado, Instituto Universitário de Lisboa). Recuperado de: http://hdl. handle.net/10071/13432

McFarland, S. (2010). Authoritarianism, social dominance, and other roots of generalized prejudice. Political Psychology, 31, 453-477. https://doi.org/10.1111/j. 1467-9221.2010.00765.x

Nicol, A. A. M., \& France, K. (2016). The Big Five's relation with the facets of Right-Wing Authoritarianism and Social Dominance Orientation. Personality and Individual Differences, 98, 320-323. https://doi.org/10.1016/j.paid.2016.04.062
O'Brien, K. S., Shovelton, H., \& Latner, J. D. (2013). Homophobia in physical education and sport: The role of physical/sporting identity and attributes, authoritarian aggression, and social dominance orientation. International Journal of Psychology, 48(5), 891-899. https://doi.org/10.1080/00207594.2012.713107

Pires, A. M. L. T. (2010). El prejuicio racial en Brasil: medidas comparativas. Psicologia \& Sociedade, 22(1), 32-42. https://doi.org/10.1590/s0102-71822010000100005

Pratto, F., Sidanius, J., Stalworth, L. M., \& Malle, B. F. (1994). Social dominance orientatio: A personality variable predicting social and political attitudes. Journal of Personality and Social Psychology, 67, 741-763. https://doi.org/10.1037/0022-3514.67.4.741

Pratto, F., Sidanius, J., \& Levin, S. (2006). Social dominance theory and the dynamics of intergroup relations: Taking stock and looking forward. European Review of Social Psychology, 17, 271-320. https://doi. org/10.1080/10463280601055772

$\mathrm{R}$ Core Team (2019). $R$ : A language and environment for statistical computing. Acesso em 26 de março de 2019, recuperado de: https://www.R-project.org/.

Santos, M. H., \& Amâncio, L. (2014). Percepção de justiça, discriminação e sexismo. Psicologia, 28(1), 63-76.

Sibley, C. G., Duckitt, J. (2010). The ideological legitimation of the status quo: Longitudinal tests of a social dominance model. Political Psychology, 31, 109-137. https://doi.org/10.1111/j.1467-9221.2009.00747.x

Sidanius, J., Levin, S., Federico, C. M., \& Pratto, F. (2001). Legitimising ideologies: A social dominance approach. In Jost J. T., \& Major B. (Eds.), The Psychology of Legitimacy (pp. 307-331). New York, NY: Cambridge University Press.

Sidanius, J., Levin, S., Liu, J., \& Pratto, F. (2000). Social dominance orientation, antiegalitarianism, and the political psychology of gender: An extension and crosscultural replication. European Journal of Social Psychology, 30, 41-67. https://doi.org/10.1002/(sici)1099-0992(200001/02)30:1\%3C41::aid-ejsp976\%3E3.0.co;2-o

Sidanius, J., Levin, S., \& Pratto, F. (1996). Consensual social dominance orientation and its correlates within the hierarchical structure of American society. International Journal of Intercultural Relations, 20, 385-408. https://doi.org/10.1016/0147-1767(96)00025-9 
Sidanius, J., \& Pratto, F. (1999). Social dominance: An intergroup theory of social hierarchy and oppression. New York, EUA: Cambridge University Press. https:// doi.org/10.1017/cbo9781139175043

Sidanius, J., Sinclair, S., \& Pratto, F. (2006). Social dominance orientation, gender, and increasing educational exposure. Journal of Applied Social Psychology, 36(7), 1640-1653. https://doi.org/10.1111/ j.0021-9029.2006.00074.x

Sirbu, A. (2017). Empatia, Personalidade e Imagens Sociais sobre Jovens em Acolhimento Residencial: o papel mediador da Dominância Social (Dissertação de mestrado, Instituto Universitário de Lisboa). Recuperado de: http://hdl.handle.net/10071/15296

Torres, A. R. R., \& Camino, L. (2013). Grupos sociais, relações intergrupais e identidade social. In L. Camino, A. R. R. Torres, M. E. O. Lima, \& M. E. Pereira (Eds.), Psicologia social: temas e teorias (pp. 513-537). Brasília, BR: Technopolitik.

Thomas, K. J., \& Napolitano, P. H. (2017). Educational privilege: the role of school context in the development of just world beliefs among Brazilian adolescents. International Journal of Psychology, 52, 106-113. https:// doi.org/10.1002/ijop.12382

Vala, J., \& Lopes, R. C. (2016). Categorização social e fatores ideológicos na dinâmica das relações intergrupais. In França, D. X. \& Lima M. E. O. (Eds), Niveis de análise e formas de intervenção em psicologia social (pp. 43-73). São Paulo, BR: Scortecci

Valencia-Moya, J. (2018). Orientación a la dominancia social de las élites en Chile. Interciencia: Revista de ciencia y tecnología de América, 43(5), 372-378.

Wachelke, J. (2017). Ideologia nas opiniões de estudantes de ensino médio sobre sucesso no trabalho. Psicologia Ciência e Profissão, 37(3), 652-668. https:// doi.org/10.1590/1982-3703004062016

Wachelke, J. (2018a). Posição social de adolescentes e a crença no mundo justo. Arquivos Brasileiros de Psicologia, 70(2), 65-80.

Wachelke, J. (2018b). Avaliação de adolescentes sobre a importância de valores para a sociedade: relações com classe econômica e escolaridade dos pais. Psychologica, 61(2), 47-68. https://doi.org/10.14195/1647-8606_61-2_3
Recebido em: 17/4/2019.

Aprovado em: 13/6/2019.

Publicado em: $\mathrm{xx} / \mathrm{x} / \mathrm{xxxx}$

Endereço para correspondência: João Wachelke (Av. Pará, 1720 - Bairro Umuarama- Campus Umuarama - Bloco 2C - Sala 2C19, Uberlândia-MG, CEP 38405 320; (34) 3225 8522; joao.wachelke@ufu.br).

\section{Nome: Joao Wachelke}

E-mail: joao.wachelke@ufu.br

Titulação Acadêmica: Doutor em Psicologia Social e da Personalidade - Università degli studi di Padova Afiliação Institucional: Professor adjunto do Instituto de Psicologia da Universidade Federal de Uberlândia (UFU)

\section{Nome: Fabíola Rodrigues Matos}

E-mail: fabiolarmatos@yahoo.com.br

Titulação Acadêmica: Mestre em Psicologia Universidade Federal de Uberlândia Afiliação Institucional: Universidade Federal do Espírito Santo 